\title{
Fuel Cell Micro-CHP Techno-Economics: Part 1 - Model Concept and Formulation
}

\author{
A.D. Hawkes ${ }^{1, a}$, D.J.L. Brett ${ }^{b}$, N.P. Brandon ${ }^{c}$ \\ ${ }^{\mathrm{a}}$ Grantham Institute for Climate Change, Imperial College London, London SW7 2AZ, UK \\ ${ }^{\mathrm{b}}$ Dept. Chemical Engineering, University College London, London WC1E 6BT, UK

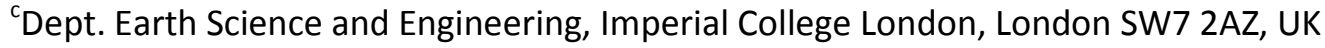

\section{Abstract}

This article presents the concept and mathematical treatment for a techno-economic modelling framework designed to enable exploration of fuel cell micro combined heat and power (micro-CHP) system design and control. The aim is to provide a tool that can help to focus research and development attention on the system characteristics critical for commercial success of these technologies, present cost targets for developers, and to ensure policy makers provide appropriate instruments to support commercialisation. The model is distinctive in that it applies mixed integer unit commitment formulation to link design and control decisions for micro-CHP, and explicitly characterises stack degradation in a techno-economic framework. It is structured to provide depiction of the fuel cell stack and balance-of-plant, supplementary thermal-only system (e.g. tail gas burner), thermal energy storage, and electrical power storage. Technically, the fuel cell stack is characterised by steady-state thermal and electrical efficiencies for full and part-load operation, its nameplate capacity, minimum operating set point, and stack degradation via performance loss rate proportional to power density and thermal cycling rate. The dynamics of operation are emulated via ramp limits, minimum up-time and minimum down-time constraints, and start-up and shutdown costs and energy consumptions. The primary performance evaluation metric adopted is the

\footnotetext{
${ }^{1}$ Corresponding author. E-mail a.hawkes@imperial.ac.uk
} 
maximum additional capital cost a rational investor would pay for the fuel cell micro-CHP system over and above what they would pay for a competing conventional heating system. The companion article (Part 2) applies the developed model to consider the impact of stack degradation on economic and environmental performance.

Keywords: micro-CHP, cogeneration, unit commitment, mixed integer, degradation

\section{Nomenclature}

Objective

Lifetimecost The operation and maintenance cost of meeting energy demand over the fuel cell's lifetime.

Decision Variables

$a_{2, t}, \ldots, a_{L, t} \quad$ Piecewise electrical output $\left(\mathrm{kW}_{\mathrm{e}} \mathrm{h}\right)$ of micro-CHP in time period $\mathrm{t}$.

$\alpha_{1, t}, \ldots, \alpha_{L, t} \quad$ State of each segment of piecewise electrical output $-0=$ not fully dispatched, $1=$ fully dispatched for each time period $t$.

$S_{s t, t} \quad$ Start-up cost for fuel cell stack in time period t.

$S_{s d, t} \quad$ Shutdown cost for micro-CHP in time period t.

$b_{t} \quad$ Thermal output $\left(\mathrm{kW}_{\mathrm{th}} \mathrm{h}\right)$ of supplementary heat-only unit in time period $\mathrm{t}$.

$c_{t} \quad$ Electricity bought $\left(\mathrm{kW}_{\mathrm{e}} \mathrm{h}\right)$ from the grid in time period $\mathrm{t}$.

$d_{t} \quad$ Electricity sold $\left(\mathrm{kW}_{\mathrm{e}} \mathrm{h}\right)$ to the grid in time period $\mathrm{t}$.

$e_{t} \quad$ Electricity used to charge electricity storage $\left(\mathrm{kW}_{\mathrm{e}} \mathrm{h}\right)$ in time period t.

$f_{t} \quad$ Electricity discharged from electricity storage $\left(\mathrm{kW}_{\mathrm{e}} \mathrm{h}\right)$ in time period $\mathrm{t}$.

$g_{t} \quad$ Thermal energy charge to thermal storage $\left(\mathrm{kW}_{\mathrm{th}} \mathrm{h}\right)$ in time period $\mathrm{t}$.

$h_{t} \quad$ Thermal energy discharged from thermal storage $\left(\mathrm{kW}_{\mathrm{th}} \mathrm{h}\right)$ in time period $\mathrm{t}$. 
$\lambda_{F C} \quad$ Lifetime of the fuel cell stack in years.

$F C_{\text {cap }} \quad$ Nameplate capacity of fuel cell stack $\left(\mathrm{kW}_{\mathrm{e}}\right)$.

$B_{\text {cap }} \quad$ Nameplate capacity of supplementary heat-only unit $\left(\mathrm{kW}_{\mathrm{th}}\right)$

$E S_{\text {cap }} \quad$ Nameplate capacity of electricity storage unit $\left(\mathrm{kW}_{\mathrm{e}} \mathrm{h}\right)$

TES $_{\text {cap }} \quad$ Nameplate capacity of thermal energy storage unit $\left(\mathrm{kW}_{\mathrm{th}} \mathrm{h}\right)$

$\zeta \quad$ Binary variable indicating if a micro-CHP is present.

$\vartheta \quad$ Binary variable indicating if electricity storage is present.

$\mu \quad$ Binary variable indicating if thermal energy storage is present.

$D_{1} \ldots, D_{L} \quad$ Breakpoints in micro-CHP piecewise linear generating cost function

$\eta_{F C, 1}, \ldots, \eta_{F C, L}$ Initial fuel cell net LHV electrical efficiency coefficients.

$\sigma_{F C, 1}, \ldots, \sigma_{F C, L}$ Fuel cell net LHV overall efficiency (heat + power) coefficients.

$\tau_{d} \quad$ Minimum down-time of fuel cell stack (no. of time periods).

$\tau_{u} \quad$ Minimum up-time of fuel cell stack (no. of time periods).

$\tau_{s} \quad$ Number of time periods in fuel cell start-up cost function.

$s_{\text {start }, \tau} \quad$ Start-up cost of micro-CHP system if started after $\tau$ time periods off $(f)$.

$S_{\text {shutdown }} \quad$ Shutdown cost of micro-CHP system (f).

$r_{\text {up }} \quad$ Maximum ramp up for micro-CHP ( $\left(\mathrm{KW}_{\mathrm{e}}\right.$ per time period).

$r_{\text {down }} \quad$ Maximum ramp down for micro- $\mathrm{CHP}(\mathrm{kW}$ er time period).

$\kappa_{\eta} \quad$ Micro-CHP electrical efficiency degradation coefficient $\left(\% / \mathrm{kW}_{\mathrm{e}} \mathrm{h} / \mathrm{kW}_{\text {installed }}\right)$.

$\varphi_{\eta} \quad$ Micro-CHP electrical efficiency degradation coefficient (\%/thermal cycle)

$\sigma_{S H U} \quad$ Supplementary heat-only unit LHV efficiency.

$u_{c h} \quad$ Electricity storage unit maximum charge rate $\left(\mathrm{kW}_{\mathrm{e}} \mathrm{h}\right.$ per time period).

$u_{\text {dis }} \quad$ Electricity storage unit maximum discharge rate $\left(\mathrm{kW}_{\mathrm{e}} \mathrm{h}\right.$ per time period).

$v_{c h} \quad$ Thermal energy storage unit maximum charge rate $\left(\mathrm{kW}_{\mathrm{th}} \mathrm{h}\right.$ per time period). 


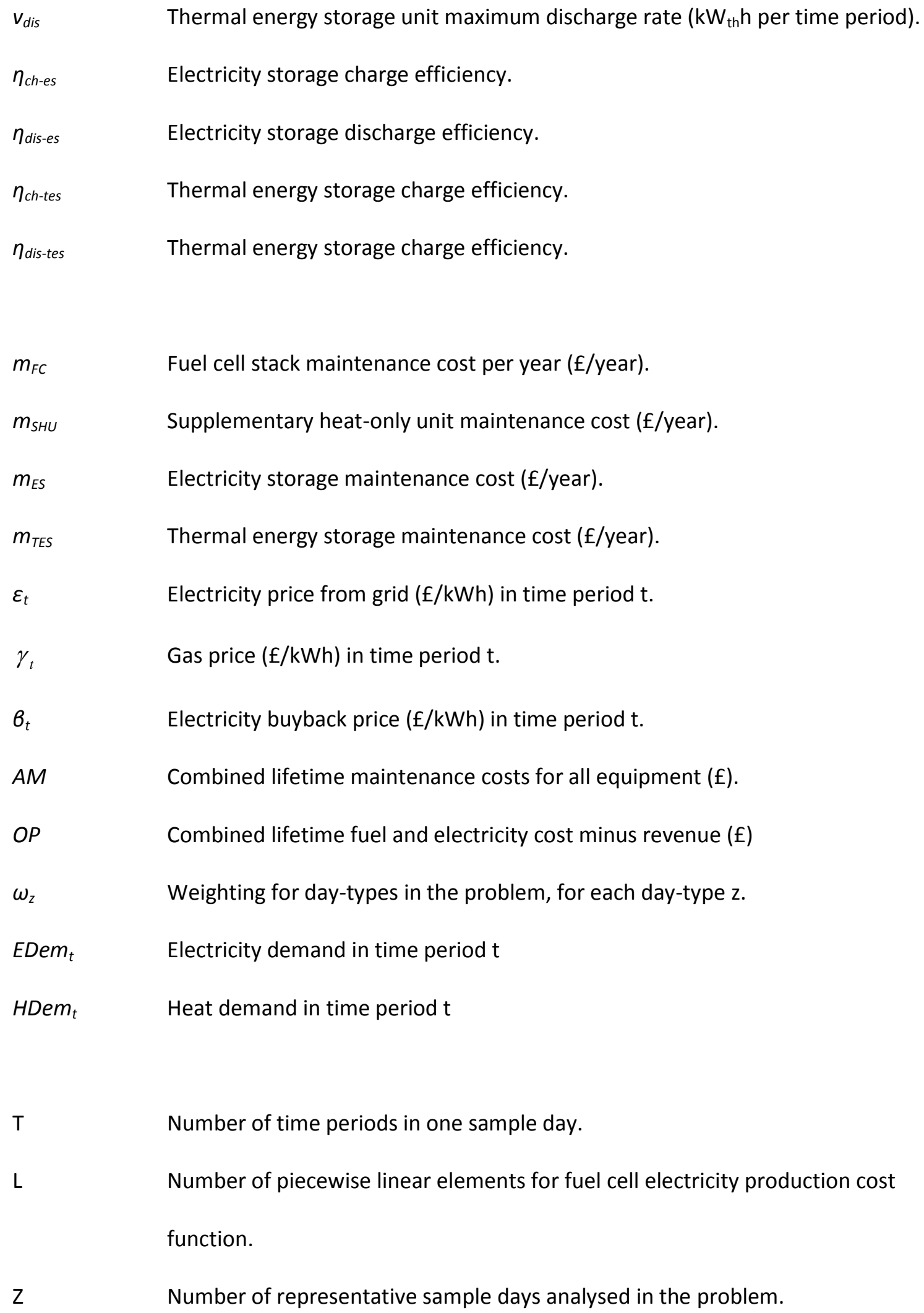
function. Number of representative sample days analysed in the problem. 


\section{Introduction}

Fuel cell based micro combined heat and power (micro-CHP) is a promising technology to efficiently meet heating and some electricity needs of residential dwellings. It has the potential to reduce carbon dioxide emissions related to energy consumption in the domestic sector, can reduce primary energy consumption which could improve national energy security, and could be a cost effective means of meeting residential energy needs if capital cost targets can be met. Furthermore, potential market size is large, suggesting the technologies could be become important in overall energy supply [1-3]. As fuel cells are an emerging technology, it is timely to provide developers and policy makers with information regarding their economic and environmental sensitivities and potential, and the relative importance of each of their technical constraints. This can aid developers in focusing research and development attention, and policy makers in formulating relevant and effective instruments to support commercialisation for the sector. A building block of most such analyses is techno-economic modelling of the systems.

This article presents the concept and develops a mathematical formulation for a techno-economic model designed to aid research and development for micro-CHP. The model applies a mixed integer linear programming methodology to minimise the cost of meeting a given electricity and heat demand using a fuel cell based micro-CHP system. This system consists of a fuel cell stack and balance-of-plant, and a supplementary heat-only unit (e.g. a condensing boiler) to help meet heat demands. The micro-CHP system is operated in parallel with the electricity grid, and as such may import electricity from the grid or export electricity to the grid. Furthermore, simple linear characterisations of electricity storage and thermal energy storage are included in the formulation. 
The unit commitment ${ }^{2}$ problem is a frequently visited area of research. Previous studies have formulated optimisation mathematics for the case of one or several generators operating in various market systems, often subject to technical constraints. A survey of recent work of relevance can be found in Padhy [4]. However, typical applications of unit commitment approaches are generally for electricity generators as opposed to combined heat and power, and are typically applied to optimal scheduling problems rather than as a tool to inform system design and control strategy as is presented here. Hawkes et al. $[5,6]$ have previously formulated non-linear programming mathematics for the case of fuel cell micro-CHP, but due to some important limitations in that approach, have developed this new model. The primary aspects that this work adds to previous research are:

- Mixed integer linear programming (MILP) unit commitment for the case of micro-CHP, with a supplementary thermal system, and thermal and electrical storage.

- Explicit characterisation of dynamic aspects of the systems, namely; start-up cost and energy use, shutdown cost and energy use, minimum up-time, minimum down-time, and turndown ratio for the case of micro-CHP.

- Degradation characterisation for the case of fuel cell based micro-CHP.

Micro-CHP systems, like most generating equipment, take some time to begin operating and incur start-up costs. This cost relates to energy consumption during start-up before the system produces useful energy. Fuel cell micro-CHP systems may incur relatively large start-up cost and may take additional time to begin operating because of dependencies between fuel processing, fuel reforming and the stack, and time taken to heat up various components in a controlled manner. Furthermore it is likely that fuel cell micro-CHP will have higher thermal mass than conventional home heating

\footnotetext{
${ }^{2}$ Optimal "unit commitment" is defined as the best dispatch strategy for each component in the system with the aim of maximising or minimising some outcome, usually profit or cost, respectively.
} 
systems, implying greater energy consumption during warm up. Therefore, modelling of these aspects of operation can lead to improved prediction of system performance and identify important system characteristics that can lead to micro-CHP products that perform better economically and environmentally. Furthermore, explicit characterisation of fuel cell stack degradation will provide system developers with better information regarding maximum installed capital cost in the target market, and understanding of the tradeoffs between system design and control to mitigate the impact of any degradation.

\section{Selected Literature Review}

Models designed to analyse the economic and environmental performance of fuel cells are becoming more common in the literature. These models can be broadly classified as either simulation or optimisation models, and as either dynamic or steady-state models. The modelling framework presented in this article is an optimisation model with steady-state technology characterisation, but dynamic qualities of system performance are captured via a set of constraints that emulate the dynamic/transient behaviour of the system, as is discussed below in the mathematical formulation. Optimisation modelling present in the literature can be further disaggregated into optimal operation modelling (i.e. unit commitment) and engineering based pinch point design ${ }^{3}$. These approaches have been applied to fuel cell CHP systems in only a handful of articles. The majority of these consider engineering design from a technical point of view, with some exceptions considering optimal operation of larger systems. These two optimisation approaches are discussed in the following two paragraphs, followed by differentiation of simulation approaches.

\footnotetext{
${ }^{3}$ Pinch point design is a methodology for calculating minimum energy consumption of a process, and attempting to achieve it via optimal design of heat exchange/recovery systems, tailoring operating parameters, and choosing energy supply methods.
} 
Unit commitment of large polymer electrolyte membrane (PEM) fuel cell systems has been investigated $[7,8]$ using evolutionary programming techniques ${ }^{4}$, although these were applied to consider performance driver rather than to explicitly consider system design. Also, cost-effective operating strategies for micro-CHP including fuel cells have been investigated using non linear programming, demonstrating that simple strategies such as following electricity demand can result in poor performance [9]. Non-linear programming (NLP) techniques have been developed for the case of solid oxide fuel cell (SOFC) micro-CHP, which simultaneously consider stack capacity (i.e. an element of system design) and operating strategy [6]. This approach was applied to consider the influence of limits on the rate of change of current density (i.e. ramp rate limits) on system economics and optimal stack sizing. However, the present article notes that a non-linear programming formulation is unable to effectively capture start-up, shutdown, minimum up-time or downtime, or maximum turndown of the system, which could all have important influences on system economics and environmental credentials.

Optimum engineering configuration of systems has been tackled using techniques such as pinch point design. Maréchal et al. [10] and Palazzi et al. [11] developed an approach where system efficiency is maximised, and installed cost is minimised using a detailed technical system representation and multi-objective optimisation. These studies assumed constant output from the fuel cell stack (a large $50 \mathrm{~kW}_{\mathrm{e}}$ ), thereby ignoring the influence of part-load operation on optimal system design. Wallmark and Alvfors [12] performed pinch point design of a $15 \mathrm{~kW}_{\mathrm{e}}$ PEM fuel cell system to arrive at an optimal steady-state representation, which was then simulated for an economic analysis of a building in Sweden. Whilst these detailed pinch point analyses are critical for refining the technical design of systems, they do not provide the feedback loop between operational strategy and system design that unit commitment can, and therefore do not provide a direct link between design and economic/environmental performance.

\footnotetext{
${ }^{4}$ Evolutionary programming techniques emulate the mutation and selection processes of biological evolution in order to converge to an optimal point.
} 
Simulation modelling approaches considering the economics of fuel cell systems are also represented in the literature, but they are distinct from optimisation-based approaches. Simulation is defined here as modelling that steps though time and predicts response and performance of the fuel cell system according to some predefined operating strategy, and possibly its interaction with the dwelling within which it is operating. These models do not optimise operation or design of the fuel cell system. Like optimisation models, simulation models vary greatly in complexity. For example, simple approaches can assume constant output from the fuel cell, and single-value steadystate efficiency characterisation (for example, [13]). More complex approaches include detailed performance characterisation, ranging from part-load steady-state efficiency characterisation (a profile over the range of output) and a fuel cell stack that is able to modulate output (for example [14]), through to more technical engineering-based dynamic simulations (for example, [15]) that typically do not consider economic or environmental credentials. The final simulation approach to be mentioned is building simulation, where thermal energy flows and interactions are endogenously characterised, enabling sophisticated thermal analysis. Only one reviewed article that applied simulation modelling included a degradation characterisation in an economic sense [16]. This model applied a constant degradation rate to a steady-state technology representation, and was employed to estimate capital cost targets for fuel cell micro-CHP in the UK market. The approach is not designed to reflect degradation issues back onto an optimisation objective (i.e. the operating strategy does not respond to the economic influence of degradation).

Within the array of modelling approaches discussed above, there is a valuable approach that is not well represented. As fuel cell micro-CHP is an emerging technology it is appropriate to provide developers with realistic information regarding their design and control decisions in terms of economic performance and environmental outcomes. This approach represents the key high-level techno-economic characteristics of fuel cells (e.g. capacity, degradation, start-up and shutdown 
performance, minimum up-time and minimum down-time, part-load efficiency), and optimises the control strategy of the system based on minimising the cost of meeting the lifetime electricity and power demand of the dwelling. Each high-level technical characteristic can then be altered in a sensitivity analysis to investigate its importance. This modelling approach can therefore be used to identify the key technical characteristics for commercially successful fuel cell micro-CHP in a way that provides a feedback loop between design decisions and operational strategy. Such an approach is developed in this article.

\section{$3 \quad$ Fuel Cell Degradation}

This section briefly describes the mechanism of degradation and how it is treated in this model. For further information including a more detailed review of degradation in the literature and estimates of degradation rates (and an application of the model presented herein) the reader is referred to the companion article [17].

Fuel cell performance is adequate for most applications. The focus is now shifting to maintaining this performance throughout its life or restricting the degradation to an acceptable level. Successful commercialisation will only be possible when both capital cost and endurance issues are addressed. The sources of fuel cell performance degradation are various mechanisms related to all main components of the stack (i.e., electrolyte, electrodes and bipolar plates) and are often specific to the type of fuel cell technology. These degradation mechanisms may be;

- reversible (e.g. flooding, flow channel blockage, electrolyte membrane dehydration in PEMFCs),

- partially reversible (e.g. poisoning by fuel impurity, SOFC anode oxidation), and

- irreversible (e.g. sintering of electro-catalyst, electrolyte decomposition, catalyst support corrosion). 
Some may be exacerbated or remedied by excursions from steady-state operation, such as the cycling of temperature, electrical load, humidification level, electrode oxidation state, etc. Degradation will always be a feature of fuel cell performance and will be a function of cell/stack design and fabrication methods, materials, operating mode and the fuel used.

For the purposes of the model formation presented in the next section, stack degradation mechanisms are assumed to produce; A) net AC electrical efficiency degradation rate proportional to power density in the stack and/or B) net AC electrical efficiency degradation rate proportional to the thermal cycling rate of the stack.

\section{$4 \quad$ Modelling Concept}

This section presents the concept behind the developed model, describing why particular performance metrics have been adopted, and outlining the inputs, outputs, and flow of the modelling approach. This argument is framed by commercial deployment pathways for fuel cell micro-CHP as follows.

\subsection{Micro-CHP Commercial Deployment Pathways}

The nature of the driving force in a micro-CHP investment decision depends largely on which stakeholder is making that decision. In order to gain understanding of appropriate performance modelling metrics, it is useful to consider which actors are associated with each potential route to market. To investigate this issue a set of deployment models have been developed in by Watson in [18] and applied to investigate social acceptance of microgeneration in Sauter and Watson [19]. These three deployment pathways are considered here to inform choice of performance metrics. They are: 
- Plug-and-play. This pathway assumes the decision to invest in microgeneration is taken independently by the dwelling owner or occupier who finances the purchase.

- Company control. This pathway involves more passive consumers that provide a site for the system which is owned and/or operated by an Energy Service Company (ESCo) or energy supplier.

- Community Microgrid. Where a group of individuals/businesses group together to provide some of their collective energy needs, and own and operate the units. The community microgrid option is beyond the scope of this article as it relates more to larger scale systems.

The plug-and-play and company control pathways are discussed in the following two sub-sections, with reference to the theory of diffusion of innovations. This theory is instructive here because it attempts to define how information about a new product spreads through a social system and the processes individual adopters go through regarding an uptake decision. The theory can therefore elucidate what factors may be important in individuals' investment decisions, aiding choice of primary performance metrics for the modelling framework.

\subsubsection{Plug-and-Play Deployment Pathway and Diffusion of Innovations}

When considering the individual private investor (i.e. owner or occupier of a dwelling for the case of micro-CHP in the plug-and-play pathway), the market is typically split into a number of "types" of investor based on their propensity for innovation. Rogers [20] classified these as innovators, early adopters, early majority, late majority, and laggards. This disaggregation, known as the Rogers Adoption Curve, is displayed in Figure 1, and leads to typical "S" penetration curves. According to Rogers the primary variables determining the rate of adoption of an innovation are;

- it's perceived attributes,

- the type of innovation decision,

- the type of communication channels, 
- the nature of the broader social system, and

- the extent of change agents' promotion efforts.

The discussion here focuses on the "perceived attributes" variable as it is the most relevant to techno-economic analysis of fuel cell micro-CHP. Within the perceived attributes category the first sub-dimension is the relative advantage of the innovation, followed by compatibility, complexity, trialability and observability ${ }^{5}$. Fuel cell micro-CHP can be considered in terms of these attributes given the fact that it is a technology designed to replace existing home heating systems such as boilers. Therefore it is reasonable to assume that it is "compatible" with lifestyle and values in many important markets, as in many respects it resembles boiler systems and performs an almost identical function. Likewise it is reasonable to assume that micro-CHP, when well designed, can be simple to use, circumventing any "complexity" barriers. Trialability and observability could be more challenging issues, but the nature of the technology suggests they are just as trialable and observable as any competing home heating systems, with the exception of the incumbent technology. However, at this stage of micro-CHP's development, it is apparent that the most important perceived attribute for micro-CHP is related to its relative advantage.

Relative advantage is the degree to which an innovation is perceived as being better than the idea it supersedes. Typically the first listed relative advantage of a product is related to initial cost or economic profitability. Therefore, as one would presume, economic elements play an important part in relative advantage, although they are certainly not the only important factor. For example, it would be expected that innovative adopters in Rogers' bell-shaped curve are less influenced by economics, and laggards may resist (or be unaware of) change despite economic advantages. Other relative advantages typically cited in the theory of diffusion of innovations are decrease in

\footnotetext{
${ }^{5}$ Readers may refer to the referenced literature for graphical representation of these categories and subcategories.
} 
discomfort, social prestige, saving time/effort, and immediacy of reward. This list of further relative advantages is not so relevant for micro-CHP which could have modest impact on comfort, little social visibility, few obvious service advantages, and little inherent immediate reward. Therefore, economic profitability, possibly combined with social prestige if "green" credentials can be marketed effectively or become visible via other channels, appear to be the main relative advantages that apply.

Specific studies considering diffusion theory and micro-CHP relevant to the plug-and-play deployment model are available in the literature, further indicating that economics will play a vital role in achieving a mass market: El Mehdi and Kunsch [21] developed a Bass ${ }^{6}$ diffusion model for micro-CHP and used it to investigate the effectiveness of incentives such as grants or other policy instruments in influencing its adoption. They found that incentives based on the price of energy or the electricity buyback price should be most effective because they engage the consumer more with their energy use than upfront grant support. Meijer et al. [24] considered the importance of various uncertainties in "take-off" of micro-CHP diffusion in the Netherlands, and concluded that resolving uncertainty regarding buyback prices and taxation arrangements was an important element of a future market.

Overall the theory of diffusion of innovations and all the reviewed applications of diffusion models to micro-CHP indicate that economic metrics are important in the plug-and-play deployment model. It may be concluded that if performance can be demonstrated, and this information is spread effectively via typical "Bass" communication channels, the economic credentials of the system will

\footnotetext{
${ }^{6}$ Similarly to Rogers, Bass [22] posited that the probability an individual will adopt an innovative product is influenced by the number of previous adopters in a social system and a constant relating to the number of innovators. The number of previous adopters becomes the primary driving force in diffusion when it reaches a significant quantity, whilst innovators are more important in the early stages where uncertainty of performance is an issue. Norton and Bass [23] extended this model to account for technology substitution, which deals with successive generations of competing products, as is arguably the case for home heating systems such as microCHP. Bass' work is frequently used to characterise technology diffusion and substitution, and is therefore probably the most important adoption/diffusion model for the case of micro-CHP.
} 
become key elements of a penetration threshold being broken, and the technology becoming massmarket. This is partly because other drivers of adoption are not so relevant for micro-CHP, which is effectively a product that will substitute existing boiler systems and offer little visibility and few service advantages in its most basic configuration. Therefore, although economic performance of micro-CHP is not the only important factor dictating an investment decision, it is certainly a critical element.

\subsubsection{Company Control Deployment Pathway}

The company control or Energy Service Company (ESCo) deployment pathway assumes a service-led approach, where a company offers to supply heat and other energy services to a dwelling, and applies micro-CHP technology to achieve this. The dwelling occupier pays for this service, and implicitly pays for the micro-CHP system as well, although direct payment of system capital costs may be avoided via leasing arrangements, etc. Avoidance of direct payment of capital costs plays well to Rogers' "low initial cost" and "immediacy of reward" relative advantages, which are identified barriers for the plug-and-play pathway discussed above. The ESCo model also complements current added-value arrangements between customers and energy suppliers, where the customer could engage the supplier to provide energy services in an ESCo-like arrangement.

The company control pathway is also arguably more effective at introducing efficiency and generally improved performance in the residential sector because it largely takes the investment decision out of the hands of the dwelling occupier, and therefore allows that decision to be more explicitly economically-driven than in the plug-and-play pathway. ESCo (or energy suppliers acting as ESCos) actors can almost always be assumed to be economically rational, making investment decisions based on classic parameters such as net present value. Therefore the success of the company control deployment pathway in allowing micro-CHP to reach a mass market can be closely linked to the economic advantage the systems offer. Likewise with the plug-and-play pathway, other factors relating to diffusion of innovations will also play a role, but economics is likely to be crucial. 


\subsubsection{The Primary Performance Metric}

Based on the above discussion it is clear that economic considerations form a primary concern in terms of achieving a mass market for micro-CHP for both plug-and-play and company control commercial deployment pathways. They are not the sole issue, but where other factors are addressed, economic profitability will be critical to achieving a large market share. Therefore system economics is chosen as the pivotal performance metric in the present modelling framework. The primary issues relating to formulating a valid economic metric for analysis are ensuring relevance to both deployment pathways and circumventing the lack of established capital cost data for micro-CHP systems ${ }^{7}$, without which calculation of typical investment metrics (such as net present value) are not possible.

As discussed in section 4.1.1, attitudes and expectations regarding performance are inextricably linked to those of current residential heating systems. The investment decision for micro-CHP can therefore be compared with that of conventional home heating systems such as boilers, and it is the capital cost difference between the two options that the potential adopter faces ${ }^{8}$. Therefore, the primary metric chosen in this article is the marginal net present value of the micro-CHP system with respect to the competing reference system. The deployment pathway this relates to is that where an ESCo or energy supplier (i.e. the company control model) provides a micro-CHP system under a leasing arrangement. The metric is determined by calculating the discounted cashflow the ESCo would obtain from operating micro-CHP in the customer's dwelling, and can hence act as a guide for the maximum marginal capital cost that ESCo would pay for the system. The metric is cost-neutral for the dwelling occupier in that it assumes gains afforded through reduced operational costs (where the cost of meeting the energy demand using micro- $\mathrm{CHP}$ are lower than the cost using the reference system) are offset exactly by the leasing cost (i.e. the annualised capital cost) of the micro-CHP

\footnotetext{
${ }^{7}$ Whilst capital cost indicators do exist in the early developing market, there is little basis to assume these will reflect the prices of mature mass market products.

${ }^{8}$ The potential adopter must purchase a home heating system, the choice only relates to whether they opt for the conventional (i.e. boiler) system, or the substitute (i.e. the micro-CHP system).
} 
equipment. Therefore it could also be interpreted as the maximum marginal capital cost a dwelling occupier would pay for outright purchase of the micro-CHP system in the plug-and-play model, if they would accept the chosen cost of capital. However, as observed costs of capital for private residential energy-related purchases vary widely, the ESCo revenue interpretation is preferred.

The chosen primary metric therefore caters directly to the company control (ESCo or energy supplier led deployment pathway), and also has relevance to the plug-and-play deployment pathway. It also avoids the issue of incorporating uncertain capital costs into economic calculations, representing the maximum allowable capital cost rather than guessing at a specific capital cost. Finally, it maintains relevance of results for micro-CHP system developers, who require targets costs for manufacturing of systems.

\subsection{A Further Performance Metric}

In addition to the primary economic metric discussed above, a further metric is required to assess the ability of micro-CHP to aid in achieving an important aim of energy policy. This relates to reduction of greenhouse gas emissions. Additional metrics of interest to policy makers (such as energy security and system-wide efficiency of investment) are discussed in the literature, for example $[9,25,26]$, and are not covered here.

For greenhouse gas emissions reduction the adopted metric is the annual $\mathrm{CO}_{2}$ savings provided by the micro-CHP system when compared to that of the competing reference system. This measure considers only the operational greenhouse gas emissions, and does not place any economic value on reductions. Indeed at present it is uncertain which actors would capture the value of the $\mathrm{CO}_{2}$ mitigated. Lifecycle emissions due to manufacturing, fuel chain, disposal/recycling are not included. For a discussion of life cycle assessment of micro-CHP systems (which does not consider system operation in detail) readers are referred to Pehnt [27]. Greenhouse gas emissions are not only a policy-related metric; they may also be important for adoption and diffusion if micro-CHP is 
marketed as a "green" product, generating relative advantages like social prestige as discussed above in section 4.1 .

The $\mathrm{CO}_{2}$ metric is calculated based on the results of the optimisation. Therefore it relates to a situation where the economic performance of the system has been optimised, leading to $\mathrm{CO}_{2}$ results; carbon dioxide concerns are therefore not the objective of the optimisation. This implies that the primary driver for micro-CHP adoption is assumed to be economic, and the influence of micro-CHP on $\mathrm{CO}_{2}$ reduction is consequential.

\subsection{Overview of the Optimisation Modelling Approach}

In order to investigate sensitivities of the primary economic metric to system design and control, optimisation is applied. Specifically, the cost of meeting the electricity and heat demand of the target dwelling using a grid-parallel fuel cell micro-CHP system is minimised (over the fuel cell stack's lifetime). This is then compared to the cost of meeting an identical demand with the reference system (in this case a condensing boiler and grid electricity). This results in the marginal value of the micro-CHP system relative to the reference system. The marginal net present value of the microCHP system can then be calculated based on the lifetime of the micro-CHP system and a given cost of capital (i.e. the primary economic metric, as discussed above). The inputs and outputs of this formulation are described in Figure 2.

The central "optimisation" box in Figure 2 forms the core computational effort of the modelling. The micro-CHP system technical inputs and energy demand profiles form the optimisation constraints, and the economic inputs and some technical micro-CHP characteristics combine to form the objective function. The complete mathematical formulation of the optimisation problem is presented below. 


\section{Mathematical Formulation}

This section presents the efficiency characterisation for the fuel cell system and supplementary boiler, and the objective function and constraints of the optimisation. Decision variables are defined in the nomenclature. The system consists of a prime mover and balance-of-plant, and a supplementary heat-only unit (e.g. a condensing boiler) to help meet heat demands. The system is grid connected, and may import electricity from the grid or export electricity to the grid. Furthermore, simple linear characterisation of electricity storage and thermal energy storage are included in the formulation.

\subsection{Degradation Efficiency Characterisation}

As noted above, fuel cell stacks experience degradation over their lifetime. This results in operating efficiencies being lower than original values as the unit progresses through its life. The following mathematical characterisation is applied to capture degradation rate proportional to power density in the stack and rate of thermal cycling. In this model degradation is characterised for the fuel cell system for electrical efficiency only. The amount of fuel used by the stack for a given electrical energy output is influenced by degradation coefficients $\mathrm{K}$ and $\phi$. These two coefficients are defined as follows:

- $\mathrm{K}$ is the efficiency degradation per $\mathrm{kWh}$ of electrical output from the fuel cell stack (i.e. degradation rate proportional to power density).

- $\phi$ is the efficiency degradation per thermal cycle of the fuel cell stack.

Both degradation coefficients are applied to give total degradation up to the specified point in time, which is then subtracted from the original efficiency of the unit. Therefore this formulation represents linear reduction of electrical efficiency of the fuel cell stack proportional to the two 
coefficients. For these two parameters, efficiency in time period $t$ is related to the original efficiency via Equation 1.

$$
\eta_{t, F C, k}=\eta_{0, F C, k}-\kappa_{\eta} \sum_{i=1}^{t}\left(D_{1} \alpha_{1, i}+\sum_{k=2}^{L} a_{k, i}\right)-\varphi_{\eta} \frac{\sum_{i=1}^{t} S_{s d, i}}{S_{s d, t}}
$$

\section{Equation 1}

However, use of Equation 1 directly results in a non-linear objective function (see definition of the objective below), so a linear approximation must be formulated to allow representation as a mixed integer linear programming (MILP) problem. An example linearisation of an element of the objective function, using a truncated Taylor series expansion, is presented in the Appendix.

\subsection{Objective Function}

The objective of this optimisation is to minimise the lifetime cost for meeting a given electricity and heat demand, represented by the following equation:

$$
\text { LifetimeCost }=A M+O P
$$

\section{Equation 2}

Lifetime cost consists of the sum of annual maintenance costs for each piece of equipment (AM), and lifetime operating energy costs (OP) made up of fuel cost for the fuel cell and supplementary heat-only unit, costs for any electricity bought from the grid, minus revenue from any electricity sales to the grid. These two quantities are formulated as per Equation 3 and Equation 4 respectively.

$$
\begin{gathered}
A M=\lambda_{F C}\left(\zeta\left(m_{F C}\right)+m_{S H U}+\vartheta\left(m_{E S}\right)+\mu\left(m_{T E S}\right)\right) \\
\text { Equation } 3 \\
O P=\sum_{y=1}^{\lambda_{F C}}\left\{\sum_{z=1}^{Z} \omega_{z}\left(\sum_{t=1}^{T}\left[\left(\sum_{k=1}^{L} \frac{a_{k, t} \gamma_{t}}{\eta_{t, F C, k}}\right)+\frac{b_{t} \gamma_{t}}{\sigma_{S H U}}+\varepsilon_{t} d_{t}+S_{s t, t}+S_{s d, t}\right]\right)\right\}
\end{gathered}
$$




\section{Equation 4}

Equation 4 sums the fuel and electricity cost (minus revenue from electricity sold) and start-up and shutdown costs over a sample day, and then weights the result according to how many days there are of that type in a year. This process is repeated for each sample day ${ }^{9}$ in the problem. The result to this point represents the annual energy cost for meeting the electricity demand. For the case of micro-CHP without degradation, this annual energy cost can be used (compared with the cost of operating the reference system) to calculate the primary economic metric. For cases where degradation is present, the entire lifetime of the system must be considered simultaneously (as opposed to only considering one year of operation) such that operating strategy decisions at the beginning of life can reflect on performance later in life.

\subsubsection{Constraints}

\subsubsection{Minimum Up-Time and Minimum Down-Time}

Generators are typically constrained to be "on" or "off" for certain minimum time periods. This is to prevent rapid cycling of a unit, which may cause damage. Fuel cell micro-CHP can be subject to this type of constraint because rapid thermal cycling can lead to degradation of the stack and balance-ofplant.

The minimum up time and minimum down time constraints are formulated as per Nowak and Romisch [28] such that they rely only on the first integer variable (i.e. on/off state) for the micro-CHP prime mover for each time period. Equation 5 and Equation 6 present minimum up time and minimum down time constraints respectively.

\footnotetext{
${ }^{9}$ A sample day is defined as energy demand profiles for a day that is chosen to represent overall characteristics of annual energy demand for the target dwelling. Typically a set of sample days would be used in an analysis to characterise all aspects of demand.
} 


$$
\alpha_{1, t-1}-\alpha_{1, t}+\alpha_{1, \tau} \geq 0 \text { for } \tau=t+1, \ldots, \min \left[t+\tau_{u}-1, T\right] \text { and } t=2, \ldots, T \text {. }
$$

\section{Equation 5}

$$
\alpha_{1, t-1}-\alpha_{1, t}+\alpha_{1, \tau} \leq 1 \text { for } \tau=t+1, \ldots, \min \left[t+\tau_{d}-1, T\right] \text { and } t=2, \ldots, T \text {. }
$$

\section{Equation 6}

Consequently both minimum up time and minimum down time can be represented with one constraint per time period except in cases where the minimum up time is greater than the minimum down time or vice versa (which requires more constraints).

\subsubsection{Micro-CHP Piecewise Electrical Dispatch Order}

In conventional MILP unit commitment problems, dispatch order of each segment of the piecewise generating cost function is often not constrained. This is because where the cost function is strictly monotonically increasing the first segment will always be used first, followed by the second segment, etc., which correctly represents the physical system. An example of this approach can be found in Carrión and Arroyo [29]. However, when the cost function is not strictly monotonically increasing (i.e. non-convex), or constraints interact with the objective function in other ways, it becomes necessary to constrain the order in which segments are used. For example, if the generator cost curve was as per Figure 3 and the dispatch order was left unconstrained, segment $a_{3}$ would be dispatched before segment $\mathrm{a}_{2}$ because its gradient is smaller. In reality the physical system requires that $a_{2}$ is completely dispatched before $a_{3}$, so it is necessary to provide constraints to ensure that this happens.

In the present case of fuel cell micro-CHP, the necessity of the thermal supply/demand constraints (i.e. the generator produces heat as well as power) requires these dispatch order constraints, regardless of convexity or non-convexity of the generator's cost function. The dispatch order constraints are formulated as per Arroyo and Conejo [30] in Equation 7, Equation 8 and Equation 9: 


$$
\left(D_{k}-D_{k-1}\right) \alpha_{k, t} \leq a_{k, t} \text { for } k=2, \ldots, L-1 \text { and } t=1, \ldots, T \text {. }
$$

\section{Equation 7}

$$
a_{k, t} \leq\left(D_{k}-D_{k-1}\right) \alpha_{k-1, t} \text { for } k=2, \ldots, L \text { and } t=1, \ldots, T \text {. }
$$

\section{Equation 8}

$$
\alpha_{k, t} \in\{0,1\}
$$

Equation 9

\subsubsection{Minimum Set Point Constraint}

Generators typically have a minimum set-point for operation, below which technical constraints such as relatively large balance-of-plant loads make operation excessively expensive or physically damaging to the system. In this formulation segment $\mathrm{a}_{1}$ (see Figure 3 ) of the generator's output is multiplied by integer $\alpha_{1}$, which when combined with the piecewise electrical dispatch order constraint forces the generator to operate no lower than the minimum set-point $D_{1}$, but allows it to switch off entirely. This constraint is achieved implicitly via Equation 8.

\subsubsection{Start-up and Shutdown Costs}

Start-up and shutdown costs are incurred by generators due to use of fuel and electricity during the start/stop process. For example, compressors or other balance-of-plant may operate as the prime mover starts, requiring a draw of electricity from storage or the electricity grid. As per conventional generators, it is expected that the start-up cost will be exponential with respect to the amount of time the generator has been off (corresponding to large start-up costs if the generator is completely cold, reducing to small values when the generator is still warm). Following Nowak and Romisch [28], start-up cost is expressed as per Equation 10. Shutdown cost is modelled as a single fixed cost incurred for a system shutdown as per Equation 11. These two equations are also subject to $S_{s t, t} \geq 0$ and $S_{s d, t} \geq 0$. 


$$
S_{s t, t} \geq S_{s t a r t, \tau}\left(\alpha_{1, t}-\sum_{m=1}^{\tau} \alpha_{1, t-m}\right) \text { for } \tau=1, \ldots, \min \left(\tau_{s}, t-1\right) \text { and } t=2, \ldots, T \text {. }
$$

\section{Equation 10}

$$
S_{\text {sd,t }} \geq S_{\text {shutdown }}\left(\alpha_{1, t-1}-\alpha_{1, t}\right) \text { for } t=2, \ldots T \text {. }
$$

\section{Equation 11}

\subsubsection{Equipment Capacity Constraints}

Each piece of equipment must not exceed its nameplate output capacity. Equation 12 constrains the micro-CHP unit, Equation 13 constrains the boiler, Equation 14 limits the amount of energy charge in the electricity storage unit, and Equation 15 limits the amount of charge in the thermal energy storage unit.

$$
\sum_{k=2}^{L} a_{k, t}+D_{1} \alpha_{1, t} \leq F C_{c a p} \text { for } t=1, \ldots, T
$$

\section{Equation 12}

$$
\begin{gathered}
b_{t} \leq B_{\text {cap }} \text { for } t=1, \ldots, T . \\
\text { Equation } 13 \\
\sum_{i=1}^{t}\left(e_{i} \eta_{\text {ch-es }}-f_{i} / \eta_{\text {dis-es }}\right) \leq E S_{\text {cap }} \text { for } t=1, \ldots, T .
\end{gathered}
$$

Equation 14

$$
\sum_{i=1}^{t}\left(g_{i} \eta_{c h-t e s}-h_{i} / \eta_{\text {dis-tes }}\right) \leq T E S_{\text {cap }} \text { for } t=1, \ldots, T \text {. }
$$

Equation 15

\subsubsection{Electricity Balance Constraint}

The onsite electricity demand must be met by a combination of power from the micro-CHP, electricity from the grid and electricity discharged from the electricity storage unit. Any electricity exported or used to charge the electricity storage unit must be subtracted from that available to meet the onsite load. This constraint relationship is presented in Equation 16. 


$$
c_{t}+f_{t}-e_{t}-d_{t}+D_{1} \alpha_{1, t}+\sum_{k=2}^{L} a_{k, t}=\text { EDem }_{t} \text { for } t=1, \ldots, T \text {. }
$$

\section{Equation 16}

\subsubsection{Heat Balance Constraint}

The onsite heat demand must be met by a combination of useful heat from the micro-CHP unit, the boiler, and discharge from the thermal energy storage (TES) unit (minus any charge to the TES). This constraint is displayed in Equation 17.

$$
b_{t}+h_{t}-g_{t}+\left(\sum_{k=2}^{L} a_{k, t}\left(\frac{\sigma_{F C, k}}{\eta_{t, F C, k}}-1\right)\right)+D_{1} \alpha_{1, t}\left(\frac{\sigma_{F C, 1}}{\eta_{t, F C, 1}}-1\right)=H_{D e m} \quad t=1, \ldots T
$$

\section{Equation 17}

\subsubsection{Micro-CHP Ramp Constraints}

The rate at which the micro-CHP can change its electrical output level may be constrained in order to reduce mechanical stress or other problems caused by thermal gradients in the prime mover, and to reduce wear and tear caused by thermal cycling. The formulation of this constraint is presented in Equation 18.

$$
r_{\text {down }} \leq \sum_{k=2}^{L} a_{k, t}-\sum_{k=2}^{L} a_{k, t-1} \leq r_{u p} \text { for } t=2, \ldots, T \text {. }
$$

\section{Equation 18}

\subsubsection{Electrical and Thermal Energy Charge and Discharge Rate Constraints}

Similarly to capacity constraints, the rate at which energy can be stored or discharged is technically limited. These constraints, on the electricity storage unit and thermal energy storage unit charge and discharge variables, are presented in Equation 19, Equation 20, Equation 21 and Equation 22.

$$
e_{t} \leq u_{c h} \text { for } t=1, \ldots, T \text {. }
$$




\section{Equation 19}

$f_{t} \leq u_{d i s}$ for $t=1, \ldots, T$.

Equation 20

$g_{t} \leq v_{c h}$ for $t=1, \ldots, T$.

Equation 21

$h_{t} \leq v_{d i s}$ for $t=1, \ldots, T$.

\section{Equation 22}

\section{Demonstration of MILP Fuel Cell Model}

The model described above has been implemented using CPLEX 10, and Microsoft Visual C++.NET

2005. This section briefly demonstrates the influence of each of the optimisation's technical characterisations/constraints on the unit commitment of a fuel cell micro-CHP system. This brief analysis serves to highlight specific technical aspects of fuel cell micro-CHP that could benefit from further detailed investigation.

Figure 4 displays the impact of each of the design/control related constraints on the cost-optimal operating strategy. Specifically, Figure 4 presents these strategies for a hypothetical 1 kWe PEMFCbased micro-CHP system (with technical specification based on [12]) operating in a large UK dwelling under a current published marginal residential energy tariff ${ }^{10}$ for a north London address. Thermal energy output from the stack is displayed, along with output from the supplementary thermal system (e.g. boiler or tail gas burner), and thermal demand ${ }^{11}$. As such, it is possible to discern when the cost-optimal operating strategy has altered due to the imposition of a particular constraint by comparison of the relevant subplot with the "No Constraints" sub-plot (top left corner of Figure 4).

\footnotetext{
${ }^{10}$ This tariff is 10.5 pence. $\mathrm{kWh}^{-1}$ pence for electricity import, and 2.6 pence. $\mathrm{kWh}^{-1}$ for gas consumption. Electricity export is rewarded at the approximate wholesale price of electricity in the UK of 4.0 pence. $\mathrm{kWh}^{-1}$.

${ }^{11}$ Thermal energy demand is chosen for this figure because it is typically closely related to the optimal operating strategy i.e. optimal operation of fuel cell micro-CHP is usually to follow thermal demand.
} 
Each subplot within Figure 4 shows the optimal operating strategy when only the specified technical characteristics/constraint is imposed in the optimisation. Constraints investigated are start/stop costs, ramp limits, maximum turndown, minimum up-time, and degradation.

It is apparent from Figure 4 that each constraint either physically prevents/constrains system operation, or creates a financial incentive for a particular operating strategy. For example:

- The "Start/Stop Cost Constraints" make it more cost-effective to avoid starting the stack between approximately 18:00hr and 24:00hrs.

- "Ramp Constraints" require the system to change output level slowly in comparison to the "No Constraints" result. They also reduce stack output between 18:00hr and 24:00hrs.

- A severe "Turndown Constraint" (i.e. no turndown permitted - on/off only at full rated power) restricts the output from the stack between 17:00hrs and 18:00hrs.

- "Minimum Up-time" constraints prevent the system operating between 18:00hrs and 24:00hrs.

- "Degradation Characterisation" has the influence of increasing the thermal output from the system between 17:00hrs and 18:00hrs, where the reduction in electrical efficiency results in more of the fuel's energy being converted to heat.

Table 1 presents the corresponding annual energy, $\mathrm{CO}_{2}$ and economic outcomes for each of the constraints investigated in Figure 4.

Whilst all constraints in Table 1 have some influence on outcomes, it is clear that degradation can have a very important influence on system economics and the potential for $\mathrm{CO}_{2}$ reduction. The electrical efficiency reduction rates investigated $\left(0.6 \% . \mathrm{MW}_{\mathrm{e}} \mathrm{h}^{-1}\right.$, and $0.5 \%$ per 1000 thermal cycles) correspond to performance loss (e.g. voltage degradation) of approximately $2 \%$ per 1000 hours operation at constant current density or per 1000 thermal cycles, which is within the observed range 
of degradation rates of existing PEMFC systems as described in [17]. Based on these indicative results it is of interest to further investigate the sensitivity of economic and $\mathrm{CO}_{2}$ related outcomes across a range of degradation rates to better understand potential for avoiding such performance loss. This investigation is performed in the companion article, providing a detailed example of application of the model presented above along with a review of fuel cell degradation literature and assessment of the range of observed degradation rates [17].

\section{Conclusions}

This article has presented the concept behind and mathematical formation for unit commitment optimisation of fuel cell micro combined heat and power, including explicit characterisation of stack efficiency degradation and emulation of dynamic behaviour such as minimum up-time, ramp limits, and start-up/shutdown costs. The theory of diffusion of innovations has been applied to define performance metrics, and a mixed integer linear programming approach used to formulate this techno-economic model. The model is unique in that it considers unit commitment for micro-CHP, characterises degradation and dynamic behaviour, and applies all this to consider system design and control decisions.

This optimisation model is distinguished from more common simulation approaches in that it considers the best operating strategy for a particular set of technological characteristics, whereas simulation approaches usually use pre-defined control strategies. Whilst a pre-defined control strategy is useful for assessing an existing well developed technology, it does not provide information regarding the best way to design or control a system. This optimisation approach overcomes this barrier, and is therefore arguably more relevant to micro-CHP which is an emerging technology. The advantage of simulation approaches, which is that they are better able to represent a system's dynamic or transient behaviour, is partially offset by the developed model's explicit 
emulation of the economic/environmental influence of system dynamics via start/stop costs and energy consumptions, minimum up-time/downtime, ramp rates, etc.

The model is therefore a valuable tool for fuel cell micro-CHP system developers to investigate the impact of various design and control decisions/constraints on the case for investment, environmental impact, and other factors relevant to the commercialisation of their technology. The model has been implemented using state-of-the-art optimisation software, and the performance impact of selected design/control related constraints has been considered, along with comparison of optimal unit commitment at beginning and end of stack life where there is stack electrical efficiency degradation. Stack degradation was found to have the most significant influence on economics and $\mathrm{CO}_{2}$ reduction, and is therefore the subject of more detailed investigation in the companion article [17].

\section{Acknowledgements}

The authors gratefully acknowledge the financial support of European Commission via the NextGenCell Project, and the United Kingdom Energy Research Council (UKERC) Microgeneration theme.

\section{References}

1. Aki, H., A. Murata, S. Yamamoto, J. Kondoh, T. Maeda, H. Yamaguchi, and I. Ishii, Penetration of residential fuel cells and $\mathrm{CO} 2$ mitigation--case studies in Japan by multi-objective models. International Journal of Hydrogen Energy, 2005. 30(9): p. 943-952.

2. Erdmann, G., Future economics of the fuel cell housing market. International Journal of Hydrogen Energy, 2003. 28(7): p. 685-694.

3. Bauen, A., D. Hart, and A. Chase, Fuel cells for distributed generation in developing countries-an analysis. International Journal of Hydrogen Energy, 2003. 28(7): p. 695-701.

4. Padhy, N.P., Unit commitment-a bibliographical survey. Power Systems, IEEE Transactions on, 2004. 19(2): p. 1196-1205.

5. Hawkes, A.D., P. Aguiar, B. Croxford, M.A. Leach, C.S. Adjiman, and N.P. Brandon, Solid oxide fuel cell micro combined heat and power system operating strategy: Options for provision of residential space and water heating. Journal of Power Sources, 2007. 164(1): p. 260-271. 
6. Hawkes, A.D., P. Aguiar, C.A. Hernandez-Aramburo, M.A. Leach, N.P. Brandon, T.C. Green, and C.S. Adjiman, Techno-economic modelling of a solid oxide fuel cell stack for micro combined heat and power. Journal of Power Sources, 2006. 156(2): p. 321-333.

7. El-Sharkh, M.Y., M. Tanrioven, A. Rahman, and M.S. Alam, Cost related sensitivity analysis for optimal operation of a grid-parallel PEM fuel cell power plant. Journal of Power Sources, 2006. 161(2): p. 1198-1207.

8. El-Sharkh, M.Y., A. Rahman, and M.S. Alam, Evolutionary programming-based methodology for economical output power from PEM fuel cell for micro-grid application. Journal of Power Sources, 2005. 139(1-2): p. 165-169.

9. Hawkes, A.D. and M.A. Leach, Cost-effective operating strategy for residential microcombined heat and power. Energy, 2007. 32(5): p. 711-723.

10. Maréchal, F., D. Favrat, F. Palazzi, and J. Godat, Thermo-economic modelling and optimisation of fuel cell systems. Fuel Cells- From Fundamentals to Systems, 2005. 5(1): p. 524.

11. Palazzi, F., N. Autissier, F.M.A. Marechal, and D. Favrat, A methodology for thermo-economic modeling and optimization of solid oxide fuel cell systems. Applied Thermal Engineering, 2007. 27(16): p. 2703-2712.

12. Wallmark, C. and P. Alvfors, Design of stationary PEFC system configurations to meet heat and power demands. Journal of Power Sources, 2002. 106(1-2): p. 83-92.

13. Alanne, K., A. Saari, V.I. Ugursal, and J. Good, The financial viability of an SOFC cogeneration system in single-family dwellings. Journal of Power Sources, 2006. 158(1): p. 403-416.

14. Ferguson, A. and V. Ismet Ugursal, Fuel cell modelling for building cogeneration applications. Journal of Power Sources, 2004. 137(1): p. 30-42.

15. Aguiar, P., C.S. Adjiman, and N.P. Brandon, Anode-supported intermediate-temperature direct internal reforming solid oxide fuel cell: II. Model-based dynamic performance and control. Journal of Power Sources, 2005. 147(1-2): p. 136-147.

16. Staffell, I., R. Green, and K. Kendall, Cost targets for domestic fuel cell CHP. Journal of Power Sources, 2008. 181(2): p. 339-349.

17. Hawkes, A.D., D.L. Brett, and N.P. Brandon, Fuel Cell Micro-CHP Techno-Economics: Part 2 Model Application to Consider the Economic and Environmental Impact of Stack Degradation. Journal of Power Sources, 2009.

18. Watson, J., Co-provision in sustainable energy systems: the case of micro-generation. Energy Policy, 2004. 32(17): p. 1981-1990.

19. Sauter, R. and J. Watson, Strategies for the deployment of micro-generation: Implications for social acceptance. Energy Policy, 2007. 35(5): p. 2770-2779.

20. Rogers, E.M., Diffusion of Innovations. 5th Edition ed. 2003, New York, NY: Free Press.

21. El Mehdi, B.M. and P.L. Kunsch, Simulation of micro-CHP diffusion by means of System Dynamics. Energy Policy, 2008. 36(7): p. 2308-2319.

22. Bass, F.M., A New Product Growth Model for Consumer Durables. Management Science, 1969. 13: p. 203-223.

23. Norton, J.A. and F.M. Bass, A Diffusion Theory Model of Adoption and Substitution for Successive Generations of High-Technology Products. Management Science, 1987. 33(9): p. 1069-1086.

24. Meijer, I.S.M., M.P. Hekkert, and J.F.M. Koppenjan, How perceived uncertainties influence transitions; the case of micro-CHP in the Netherlands. Technological Forecasting and Social Change, 2007. 74(4): p. 519-537.

25. Hawkes, A.D. and M.A. Leach, The Capacity Credit of Micro Combined Heat and Power. Energy Policy, 2008. 36(4): p. 1457-1469.

26. Peacock, A.D. and M. Newborough, Controlling micro-CHP systems to modulate electrical load profiles. Energy, 2007. 32(7): p. 1093-1103. 
27. Pehnt, M., Environmental impacts of distributed energy systems--The case of micro cogeneration. Environmental Science \& Policy, 2008. 11(1): p. 25-37.

28. Nowak, M.P. and W. Romisch, Stochastic Lagrangian Relaxation Applied to Power Scheduling in a Hydro-Thermal System Under Uncertainty. Annals of Operations Research, 2000. 100: p. 251-272.

29. Carrión, M. and J.M. Arroyo, A Computationally Efficient Mixed-Integer Linear Formulation for the Thermal Commitment Problem. IEEE Transactions on Power Systems, 2006. 21(3): p. 1371-1378.

30. Arroyo, J.M. and A.J. Conejo, Optimal Response of a Thermal Unit to an Electricity Spot Market. IEEE Transactions on Power Systems, 2000. 15(3): p. 1098-1104.

\section{Appendix}

This appendix presents the method for linearisation of aspects of this problem influenced by degradation of electrical efficiency, and subsequent implications for the optimisation technique applied in this study.

In order to calculate the amount of fuel consumed by the fuel cell stack for a given electricity output in a time period, it is necessary to reduce the electrical efficiency of the system proportional to the cumulative electricity output in previous time periods. This creates a linear relationship between electrical efficiency degradation and cumulative use, but a non-linear relationship between fuel cost and cumulative use (the present optimisation requires this relationship to be linear).

The fuel consumption for each segment of the piecewise linear production curve, in time period $t$ is given by:

$$
y_{k, t}=\frac{a_{k, t}}{\eta_{F C, k}-\kappa_{\eta} \sum_{i=1}^{t-1}\left(\sum_{j=1}^{L} a_{j, i}\right)}
$$


It is possible to make a first order linear approximation of (Eq. A1), which is valid for a small region around the vector $a=a^{*}$, where $a^{*}$ is the best guess at the final optimised value of decision variables $a$. This approximation takes the form of a truncated Taylor series;

$$
y(a) \approx y\left(a^{*}\right)+J \cdot\left[a-a^{*}\right]
$$

(Eq. A2)

where $J$ is the Jacobian. The terms of the Jacobian can be calculated using the chain rule:

$$
\frac{\partial y}{\partial a_{k, i}}=\frac{\kappa_{\eta} a_{k, t}}{\left(\eta_{F C, k}-\kappa_{\eta} \sum_{i=1}^{t-1}\left(\sum_{j=1}^{L} a_{j, i}\right)\right)^{2}} \text { for all } i \neq t
$$

(Eq. A3)

$$
\frac{\partial y}{\partial a_{k, i}}=\frac{1}{\eta_{F C, k}-\kappa \sum_{i=1}^{t-1}\left(\sum_{j=1}^{L} a_{j, i}\right)} \text { for } i=t
$$

(Eq. A4)

Substituting (Eq. A3) and (Eq. A4) into (Eq. A2) results in (Eq. A5).

$$
y_{k, t} \approx \frac{a_{k, t}^{*}}{\eta_{F C, k}-\kappa_{\eta} \sum_{i=1}^{t-1}\left(\sum_{j=1}^{L} a_{j, i}^{*}\right)}+\frac{\kappa_{\eta} a_{k, t}^{*}\left(\sum_{i=1}^{t-1} \sum_{j=1}^{L}\left(a_{j, i}-a_{j, i}^{*}\right)\right)}{\left(\eta_{F C, k}-\kappa_{\eta} \sum_{i=1}^{t-1}\left(\sum_{j=1}^{L} a_{j, i}^{*}\right)\right)^{2}}+\frac{\left(a_{k, t}-a_{k, t}^{*}\right)}{\eta_{F C, k}-\kappa_{\eta} \sum_{i=1}^{t-1}\left(\sum_{j=1}^{L} a_{j, i}^{*}\right)}
$$

(Eq. A5)

(Eq. A5) can be simplified to arrive at (Eq. A6). 


$$
y_{k, t} \approx \frac{\kappa_{\eta} a_{k, t}^{*}\left(\sum_{i=1}^{t-1} \sum_{j=1}^{L}\left(a_{j, i}-a_{j, i}^{*}\right)\right)}{\left(\eta_{F C, k}-\kappa_{\eta} \sum_{i=1}^{t-1}\left(\sum_{j=1}^{L} a_{j, i}^{*}\right)\right)^{2}}+\frac{a_{k, t}}{\eta_{F C, k}-\kappa_{\eta} \sum_{i=1}^{t-1}\left(\sum_{j=1}^{L} a_{j, i}^{*}\right)}
$$

\section{(Eq. A6)}

This linearisation of degradation represents a good approximation subject to the condition $\eta_{F C, k} / \kappa_{\eta}-\sum_{i=1}^{t-1}\left(\sum_{j=1}^{L} a_{j, i}\right)>>0$. Essentially this means that for small values of degradation as would be expected in a commercial product, and for micro-CHP operating lifetimes up to a target of 40,000 hours, this linearisation represents a reasonably accurate approximation. The primary drawback of using this method is the requirement for iterative optimisation solution, substantially increasing the amount of time required to complete the optimisation.

Note that the method applied in this linearisation is used for all variables influenced by degradation in the present optimisation problem. Derivation of the linearisation does not vary significantly from that presented in this Appendix for any of these cases. 